\title{
Solar Sail - Fresnel Zone Plate Lens for a Large Space Based Telescope
}

\author{
J.T. Early
}

This article was submitted to $3^{\text {td }}$ American Institute of Aeronautics and Astronautics Gossamer Spacecraft Forum, Denver, CO, April 22-25, 2002

\section{February 13, 2002}

U.S. Department of Energy

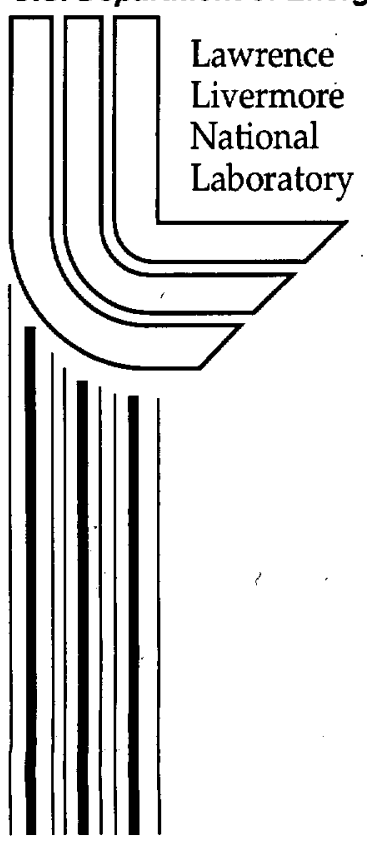




\section{DISCLAIMER}

This document was prepared as an account of work sponsored by an agency of the United States Government. Neither the United States Government nor the University of California nor any of their employees, makes any warranty, express or implied, or assumes any legal liability or responsibility for the accuracy, completeness, or usefulness of any information, apparatus, product, or process disclosed, or represents that its use would not infringe privately owned rights. Reference herein to any specific commercial product, process, or service by trade name, trademark, manufacturer, or otherwise, does not necessarily constitute or imply its endorsement, recommendation, or favoring by the United States Government or the University of California. The views and opinions of authors expressed herein do not necessarily state or reflect those of the United States Government or the University of California, and shall not be used for advertising or product endorsement purposes.

This is a preprint of a paper intended for publication in a journal or proceedings. Since changes may be made before publication, this preprint is made available with the understanding that it will not be cited or reproduced without the permission of the author.

This report has been reproduced directly from the best available copy.

Available electronically at http://www.doe.gov/bridge

Available.for a processing fee to U.S. Department of Energy

and its contractors in paper from

U.S. Department of Energy

Office of Scientific and Technical Information

P.O. Box 62

Oak Ridge, TN 37831-0062

Telephone: (865) 576-8401

Facsimile: (865) 576-5728

E-mail: reports@adonis.osti.gov

Available for the sale to the public from

U.S. Department of Commerce

National Technical Information Service

5285 Port Royal Road

Springfield, VA 22161

Telephone: (800) 553-6847

Facsimile: (703) 605-6900

E-mail: orders@ntis.fedworld.gov

Online ordering: http://www.ntis.gov/ordering.htm

OR

Lawrence Livermore National Laboratory

Technical Information Department's Digital Library

http://www.llnl.gov/tid/Library.html 


\title{
Solar sail - Fresnel zone plate lens for a large space based telescope
}

\author{
James T. Early* \\ Lawrence Livermore National Laboratory, Livermore California 94550
}

\begin{abstract}
A Fresnel zone plat lens made with solar sail material could be used as the primary optic for a very large aperture telescope on deep space probes propelled by solar sails. The large aperture telescope capability could enable significant science on fly-by missions to the asteroids, Pluto, Kuiper belt or the Oort cloud and could also enable meaningful interstellar fly-by missions for laser propelled sails. This type of lens may also have some potential for laser communications and as a solar concentrator. The techniques for fabrication of meter size and larger Fresnel phase plate optics are under development at LLNL, and we are extending this technology to amplitude zone plates made from sail materials. Corrector optics to greatly extend the bandwidth of these Fresnel optics will be demonstrated in the future.

This novel telescope concept will require new understanding of the fabrication, deployment and control of gossamer space structures. It will also require new materials technology for fabricating these optics and understanding their long term stability in a space environment.
\end{abstract}

\section{Nomenclature}

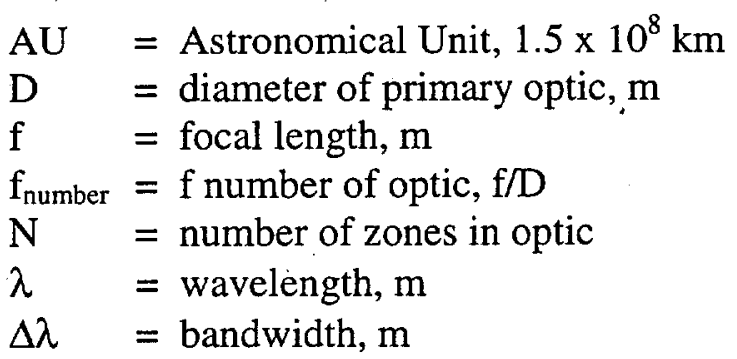

\section{Introduction}

The concept of using amplitude zone plate optics as part of sail systems has been suggested by several authors ${ }^{1,2}$. The primary issues with amplitude zone plates are the extremely narrow frequency range of these optics and the difficulties in fabricating and deploying these as large structures in space. In proposals where the zone plate is used to transmit narrow frequency laser power ${ }^{1}$, the narrow bandwidth has not been an issue. In applications where the optic is to be used as part of a telescope, only around $10^{-5}$ to $10^{-6}$ of the optical energy in a narrow frequency band can be focused into an image.

This article addresses the limited frequency response of an amplitude zone plate by the use of a corrective optic that will broaden the frequency response of the telescope by three or four orders of magnitude. This broadening will dramatically increase the optical power capabilities of the system and will allow some spectroscopy studies over a limited range. Similar corrector optics for Fresnel phase plate optical systems have already been demonstrated at LLNL ${ }^{3,4}$.

A key feature of this concept will be the use of a transmissive primary optic in the telescope design. The problem with membrane mirrors, of course, is that it is hard to control their shape to the $\lambda / 20$ scale precision needed to attain $\lambda / 10$ optical tolerances. The source of this difficultly is that mirrors reflect light, thereby doubling the effect of surface errors; in contrast, path-length errors for a thin transmissive film are canceled (not reinforced) as light arrives at

* Dep. Assoc. Program Leader, NIF Directorate, P.O.Box 808. Member AIAA 
and then departs from the surface. In actual lenses, this path-length cancellation is not perfect because the incoming and outgoing rays are not traveling in quite the same directions. If the light is bent through angle $\theta$, the $\mathrm{n}$ the path-length error in a mirror is amplified by a factor $(1+$ $\cos \theta)$, whereas that in a thin lens is reduced by $(1-\cos \theta)$. Lenses therefore have a

$(1+\cos \theta) /(1-\cos \theta)$ advantage over mirrors. By making the lens weak, i.e., by keeping $\theta$ small, this tolerance gain becomes huge. In a very slow lens (f/100) visible-light tolerances increase up to nearly a centimeter, a tremendous practical advantage when trying to field a thin opticalquality membrane optic in space.

This article will also address the issue of fabrication of large meter scale optics utilizing techniques that can be scaled to much larger sizes. At LLNL we have facilities that were built for fabrication of meter scale diffractive optics that are needed for the new laser fusion laboratory currently under construction. We now have facilities, techniques and experience personnel needed to produce very large (up to one meter in a single piece) diffractive optics in large numbers for use as diffraction gratings, Fresnel phase plate lens or optical control elements. We have also addressed issues of joining meter sized optics together to fabricate much large optical structures. This basic capability can now be used to address the issues of design and fabrication of amplitude zone plates in sail materials.

\section{Diffractive Optics Theory}

The low payload capacity of solar sail spacecraft makes the inclusion of very large aperture imaging impossible unless the optics are extremely light. If the solar sail itself forms the primary optic, then very large aperture imaging systems may be possible. The solar sail must be reflective (or absorbing for carbon sails) and very thin. If the sail is used as a reflective optic, then the $\lambda / 10$ optical precision requirement in a membrane structure will be extraordinarily difficult or impossible. The use of the sail as a transmissive optic greatly relieves the optical precision requirements, but one cannot make a transmissive Fresnel phase plate from this opaque material. The use of an amplitude zone plate optical design allows the use of reflective or absorbing sail material while still gaining the advantages of a transmissive membrane design.

The classic circular amplitude zone plate is illustrated in Fig. 1. The total number of rings, N, are given by:

$$
N=D^{2} / 8 \lambda f=D / 8 \lambda f_{\text {number }}
$$

Where $\lambda, D$ and $f$ are the wavelength, optic diameter and optic focal length respectively. The width of the outer zone (the smallest zone) is $4 \lambda \mathrm{f}_{\text {number }}$. When the large $\mathrm{f}_{\text {number }}$ is very large, then these features become easy to fabricate using optical lithography technology.

For a given optic the focal length will depend on the wavelength and will severely limit the useful bandwidth of a telescope. For good images:

$$
\Delta \lambda / \lambda<1 / 8 \mathrm{~N}=\mathrm{f}_{\text {number }} \lambda / \mathrm{D}
$$


If the $\mathrm{f}_{\text {number }}$ of the telescope is 100 and the diameter is $100 \mathrm{~m}$, then for $1 \mu \mathrm{m}$ light $\Delta \lambda / \lambda=10^{-6}$. Unless the telescope features extraordinarily large f-numbers, the zone plate primary optics will be inherently micro-bandwidth optical elements. The bandwidth may be acceptable for laser communications or power beaming, but it is a major limitation for an imaging system. For an imaging system we must achieve higher optical bandwidth by the use of chromatic correction optics.

\section{Corrector Plate for Wavelength Aberrations}

To achieve high-precision chromatic correction for the zone plate, we try to cancel its chromatic aberrations with correcting optics in the optical train of the telescope. A technique was invented 100 years ago by Schupmann ${ }^{5}$ who showed that any chromatic dispersion introduced at one optical element could be canceled by placing a second, inverse-power element with the same dispersion at an image site of the original element. The corrector optic's effectiveness will depend on its size, but increasing the bandwidth of the telescope by four orders of magnitude should be possible.

At LLNL we have fabricated Fresnel phase plates at diameters of 20 and $50 \mathrm{~cm}$. Using a corrector plate fabricated by JPL, we have demonstrated that the chromatic aberrations can be corrected in telescope configurations and that diffractions limited images can be formed with broadband $(\Delta \lambda / \lambda \sim 0.1)$ light. Both phase plates and amplitude plates have the same wavelength dependence in their chromatic aberrations. We expect to demonstrate the same effective corrections with telescopes incorporating amplitude zone plates.

\section{Amplitude Lens Demonstration Plans}

For a $f_{\text {number }} 100$ optic the outer (thinnest) ring is around $100 \mu \mathrm{m}$ for a visible optic. Fabricating the rings to a fraction of this dimension with standard optical lithography has been demonstrated with the LLNL equipment. Holding this in-plane precision in a deployed optic will depend on the material properties and the thermal control of the optic. If the optic is held at uniform temperature, then the only impact of a change in temperature is an easily corrected change in focus ${ }^{4}$.

The optic can be held flat by spinning (the preferred method) or by applying radial tension at the circumference with external structures. For transmissive optics the out of plane optical tolerances are large and easily achieved. The stresses must however be distributed without causing local in-plane distortions in the optic. If the solar sail consists of metal deposits on plastic, then the difference in thermal expansion coefficients cannot be allowed to distort the lens. If the lens consists of a uniform reflective or absorptive material, then the issue is the stress patterns created by the openings.

For a circular zone plate there will need to be structural ribs between the zones. The spacing to minimize distortions will be studied in this proposal. An alternate structure is to use orthogonal linear zone plates (Fig. 2). A zone plate consisting of straight lines can be used like a cylindrical lens to form a line focus. Two orthogonal cylindrical lens can be used as a good 
approximation of a spherical lens to form a spot focus. Similarly orthogonal linear zone plate patterns on the same optic will give a spot focus. The structural properties of this configuration will be very good, but the optical efficiency will be less than a circular zone plate.

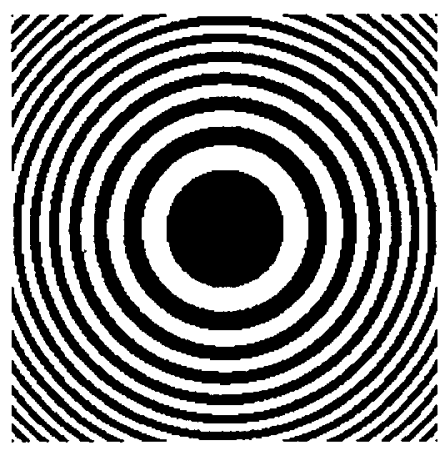

Fig. 1 - Circular Zone Plate



a



b.

Fig. 2 - Linear zone plate: a) line focus

b) point focus with orthogonal lines

\section{Solar Sail Mission Impact: Enabling for Fly-by Missions}

For solar sails to be used in actual near and/or medium term missions, they must address requirements that are not easily satisfied with conventional rocket propulsion systems. This constrains potential sail mission opportunities to high $\Delta \mathrm{v}$ missions where conventional rocket systems are very expensive and have small payloads. For inner solar system missions a solar sail can be used for rendezvous or orbiting missions, but there are relatively few inner system mission opportunities. For outer solar system missions the solar sail becomes less effective at rendezvous or orbiting because of the low solar flux at the target.

There are numerous targets for high velocity fly-by missions in the outer solar system that potentially could be served with solar sail propulsion. The basic problem is that fly-by missions in the outer solar system have low scientific data returns due to the conditions at encounter. Any imaging systems will have very little time to take data, and the data is of low resolution. First the encounter relative velocity must be high. To reach outer system targets in reasonable times the outbound leg of the trajectory is highly elliptical. The velocity of the spacecraft is typically over $10-20 \mathrm{~km} / \mathrm{sec}(2-4 \mathrm{AU} / \mathrm{y})$ relative to any target in a circular orbit ${ }^{6}$. Secondly with the exception of missions to Neptune or Uranus, the targets are typically small. Pluto and some satellites of the outer planets and potentially a few deep objects are of the order of $1000 \mathrm{~km}$ in diameter. Most asteroids, comets, Kuiper belt objects and Oort cloud objects will be under $100 \mathrm{~km}$. To get any meaningful understanding of these objects we will want resolutions better than $1 \mathrm{~km}$. For deep space missions the problem is further exacerbated by the very low light levels that will push imaging systems to longer exposure times.

With the light payload capacities of deep space missions the typical imaging system will have an aperture of $10 \mathrm{~cm}$ or less ${ }^{6}$. Table 1 gives the resolution of such a system for visible light as a function of range to target. Also listed is the time the target resolution exceeds a given value assuming the closing velocity is only $10 \mathrm{~km} / \mathrm{sec}$. It can be seen that there is only a few hours 
of moderate resolution data and very little data with resolutions better than one kilometer. In this table it is assumed that the spacecraft has maneuvered close to the target and that the separation distance is primarily along the flight path. If the camera exposure times must be long due to low illumination levels, then the high resolution data could be quite limited.

If a large fraction of the solar sail is converted into a primary optic, then the imaging system capabilities can be dramatically different. If a $100 \mathrm{~m}$ optic is part of a $200 \mathrm{~m}$ diameter solar sail, then the imaging times and resolutions are given in Table 1 . The long observation times and high resolution would enable significant imaging science. High resolution imagery of both hemispheres would be possible for even targets with low rotation rates. Dynamic events such as orbiting satellites could also be observed.

$10 \mathrm{~cm}$ optics

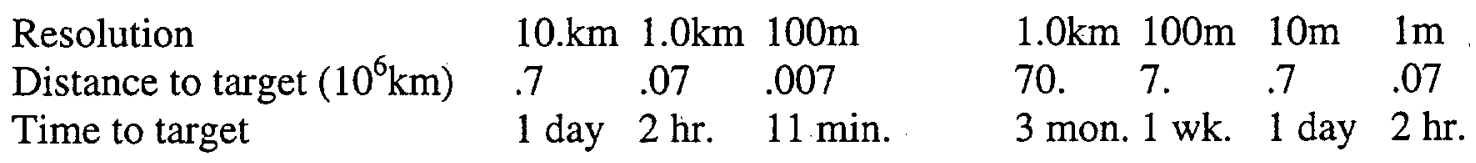

Table 1 - Imaging system performance assuming close fly-by at $10 \mathrm{~km} / \mathrm{sec}$

For missions to the asteroid belt, Kuiper belt or Oort cloud the large solar sail optic would enable fly-by missions without the spacecraft being required to maneuver to the target. For these fields of small distributed targets the spacecraft would simply fly on a straight trajectory and targets would be imaged as they passed into range. The factor of 1000 increase in optic diameter would give a factor of 1000 increase in the range at which targets could be resolved. For example if a $100 \mathrm{~m}$ optic was flown on a straight path through the Kuiper belt at $20 \mathrm{~km} / \mathrm{sec}$, in ten years based on current estimates of the belt population ${ }^{6}$, it would be able to resolve hundreds of objects including approximately 100 objects with a 1000 line resolution across the image of the object.

One objective of the Gossamer Spacecraft Initiative for solar sails is to act as a precursor mission for laser driven lightsail for interstellar missions. While there are some speculative investigations on how to slow down a lightsail at the target star system, the basic concept of the lightsail is for a fly-by mission at speeds of $0.1 \mathrm{c}$. The fundamental flaw in this mission concept is the high fly-by velocity's impact on data collection. While the target planets may be 10,000 $\mathrm{km}$ in diameter, Table 2 shows the limited observation times available at $0.1 \mathrm{c}$. Laser lightsail sizes are quite large ${ }^{7}$, so a one kilometer zone plate optic is assumed. The launch telescope for an interstellar lightsail must be around $100 \mathrm{~km}$. The use of this telescope for direct observation from the Solar System would provide resolutions near $1000 \mathrm{~km}$ in the target system which are as good as the small optic fly-by. Very large optics are required to make the fly-by mission useful. If the optics investigated by this study are shown to be feasible, then the argument of using solar sails as precursors for interstellar or outer Solar System missions will be valid. 
$10 \mathrm{~cm}$ optics

Resolution (km)

Distance to target $\left(10^{8} \mathrm{~km}\right)$

Time to target
$1 \mathrm{~km}$ optics

$\begin{array}{lllll}1000 & 100 & 10 & 1 & 0.1 \\ 7000 & 700 & 70 & 7 & 0.7 \\ 7 \text { mon. } 3 \text { wk. } & 2 \text { day } & 5 \mathrm{hr} & 30 \mathrm{~min}\end{array}$

Table 2 - Imaging system performance assuming fly-by at $0.1 \mathrm{c}$

\section{References}

1. R.L. Forward, "Roundtrip interstellar travel using laser-pushed lightsails," J. Spacecraft \& Rockets 21, 187-195 (1984).

2. Y.M. Chesnokov and A.S. Vasileisky, "Space-based very high resolution telescope based on amplitude zoned plate," presented at the International Conference on Space Optics, Toulouse Labege, France, 2-4 December 1997.

3. R. A. Hyde, "Eyeglass: Very large aperture diffractive telescopes," Applied Optics 38, No.19, 4198-4212, (1 July 1999).

4. R. A. Hyde, "Eyeglass: A Large Aperture Space Telescope," internal LLNL final report on LDRD Exploratory Research Project: 97-ERD-060

5. L Schupmann, Die Medial Fernrohre: Eine neue Konstruktion for grosse astronomisch Instrumente (B.G. Teubner, Leipzig, 1899)

6. P.K. Henry, R.J. Terrile, R.W. Maddock, and H.R. Sobel, "Exploring the Kuiper belt: an extended Pluto mission," $2^{\text {nd }}$ IAA Symposium on Realistic Near Term Advanced Scientific Space Missions: Missions to the Outer Solar System and Beyond, Aosta, Italy, June 29 - July 1, 1998.

7. G.A. Landis, "Beamed Energy Propulsion for Practicle Interstellar Flight," J. British Interplanetary Society 52, 420-423, (1999).

This work was performed under the auspices of the U.S. Department of Energy by the University of California, Lawrence Livermore National Laboratory under Contract No. W-7405-Eng-48. 